TITLE:

\title{
Discrimination of singleton and periodic attractors in Boolean networks
}

AUTHOR(S):

Cheng, Xiaoqing; Tamura, Takeyuki; Ching, Wai-Ki; Akutsu, Tatsuya

\section{CITATION:}

Cheng, Xiaoqing ... [et al]. Discrimination of singleton and periodic attractors in Boolean networks. Automatica 2017, 84: 205-213

ISSUE DATE:

2017-10

URL:

http://hdl.handle.net/2433/235515

RIGHT:

(c) 2017. This manuscript version is made available under the CC-BY-NC-ND 4.0 license

http://creativecommons.org/licenses/by-nc-nd/4.0/; The full-text file will be made open to the public on 01 October 2019 in accordance with publisher's 'Terms and Conditions for Self-Archiving'; この論文は出版社版でありません。引用 の際には出版社版をご確認じ利用ください。; This is not the published version. Please cite only the published version. 


\title{
Discrimination of singleton and periodic attractors in Boolean networks
}

\author{
Xiaoqing Cheng ${ }^{\mathrm{a}}$, Takeyuki Tamura ${ }^{\mathrm{b}}$, Wai-Ki Ching ${ }^{\mathrm{c}, \mathrm{d}, \mathrm{e}}$, Tatsuya Akutsu ${ }^{\mathrm{b}}$ \\ a School of Mathematics and Statistics, Xi'an Jiaotong University, Xi'an 710049, China. \\ ${ }^{\mathrm{b}}$ Bioinformatics Center, Institute for Chemical Research, Kyoto University, Gokasho, Uji, Kyoto 611-0011, Japan. \\ ${ }^{\mathrm{c}}$ Advanced Modeling and Applied Computing Laboratory, Department of Mathematics, The University of Hong Kong, \\ Pokfulam Road, Hong Kong. \\ ${ }^{\mathrm{d}}$ Hughes Hall, Wollaston Road, Cambridge, U.K. \\ e School of Economics and Management, Beijing University of Chemical Technology, North Third Ring Road, Beijing, China.
}

\begin{abstract}
Determining the minimum number of sensor nodes to observe the internal state of the whole system is important in analysis of complex networks. However, existing studies suggest that a large number of sensor nodes are needed to know the whole internal state. In this paper, we focus on identification of a small set of sensor nodes to discriminate statically and periodically steady states using the Boolean network model where steady states are often considered to correspond to cell types. In other words, we seek a minimum set of nodes to discriminate singleton and periodic attractors. We prove that one node is not necessarily enough but two nodes are always enough to discriminate two periodic attractors by using the Chinese remainder theorem. Based on this, we present an algorithm to determine the minimum number of nodes to discriminate all given attractors. We also present a much more efficient algorithm to discriminate singleton attractors. The results of computational experiments suggest that attractors in realistic Boolean networks can be discriminated by observing the states of only a small number of nodes.
\end{abstract}

Key words: Boolean networks, Boolean logic, attractors, observability, discrimination, biomarkers.

\section{Introduction}

Knowing internal states of complex systems is important for diagnosing various kinds of artificial, social, and biological systems. It is particularly important to identify a small set of variables so that we can reconstruct the

\footnotetext{
^ This work was partially supported by the International Collaborative Research Program of Institute for Chemical Research, Kyoto University. TA was partially supported by Grants-in-Aid 26240034 and 26540125 from JSPS, Japan, TT was partially supported by Grants-in-Aid 25730005 and 25250028 from JSPS, Japan. WKC was partially supported by Research Grants Council of Hong Kong under grant number 17301214 and National Natural Science Foundation of China under grant number 11671158. Corresponding author T. Akutsu. Tel. +81-774-38-3015. Fax. +81-774-38-3022.

Email addresses: xiaoqing9054@xjtu.edu.cn (Xiaoqing Cheng), tamura@kuicr.kyoto-u.ac.jp (Takeyuki

Tamura), wching@hku.hk (Wai-Ki Ching),

takutsu@kuicr.kyoto-u.ac.jp (Tatsuya Akutsu).
}

system's complete internal state at any given time step from time-series data of these variables. In such a case, the system is called observable. Recent studies on complex networks revealed relationships between network properties and the number of sensor nodes corresponding to those variables (Liu, Slotine, \& Barabàsi, 2013; Yan, Tsekenis, Barzel, Liu, Slotine, \& Barabàsi, 2015). However, existing studies focus on linear systems and certain types of nonlinear systems. Since biological systems contain highly nonlinear switch-like components, observability studies should also be done on systems with discrete components.

The Boolean network (BN) is known as a discrete mathematical model of gene regulatory networks (Kauffman, 1993) and has been applied to modeling of various biological systems (Albert \& Thakar, 2014). In a BN, each node corresponds to a gene and takes one of the two values 0 and 1 , where 0 (resp., 1 ) means that the corresponding gene is inactive (resp., active). The value of a node at a given time step is determined according to 
a regulation rule represented by a set of Boolean functions. Although there exist several variants, in a widely used model, the values of network nodes are updated synchronously by using the Boolean functions and the (global) state of a network at a given time step is the vector of its node values. Beginning with any initial state, the system eventually falls into an attractor, which is classified into two types: a singleton attractor corresponding to a stable state and a periodic attractor corresponding to a sequence of states that repeats periodically. Attractors are often considered as cell types: different attractors correspond to different cell types (Kauffman, 1993). Based on this interpretation, extensive studies have been done on the distribution and length of attractors in BNs (Samuelsson \& Troein, 2003; Kauffman, Peterson, Samuelsson, \& Troein, 2004; Drossel, Mihaljev, \& Greil, 2005).

Although attractors in synchronous BNs are either singleton or periodic, attractors in asynchronous BNs are more complex. An attractor in an asynchronous BN is a strongly connected component without outgoing edges in a state transition diagram (Saadatpour, Albert, \& Albert, 2010), which is also called a loose attractor (Harvey \& Bossomaier, 1997), a complex loop (Garg, Di Cara, Xenarios, Mendoza, \& De Micheli, 2008). and a terminal strongly connected component (Fauré, Naldi, Chaouiya, $\&$ Thieffry, 2006). If we consider non-linear systems obtained by extending BNs, steady states may include intermediate states (Mochizuki, 2008). Since such complex attractors or complex steady states cannot be represented as sequences of $0-1$ vectors and thus are very difficult to handle, we focus on synchronous BNs in this paper.

Recently, observability of BNs has also been studied (Cheng, Qi, \& Li, 2011; Laschov, Margaliot, \& Even, 2015; Li, Yang, \& Chu, 2015). However, due to its high nonlinearity, it is impossible in most cases to observe complete internal states of BNs from a small number of nodes. For example, Li et al. showed that more than half of the nodes are required to guarantee the observability of internal states of all attractors in the Drosophila segment polarity network ( $\mathrm{Li}$ et al., 2015). Therefore, we need to consider another approach for distinguishing the internal states of BNs.

We note here that it is important for medical diagnosis to identify the type of each cell by observing expression patterns of a few genes (e.g., biomarkers or marker genes). Therefore, extensive studies have been done to find a small number of marker genes so that disease types or cell types can be discriminated by observing expression of these genes (Whitfield, George, Grant, \& Perou, 2006). Furthermore, both gene expression data and network structure data are utilized to find more reliable marker genes (Hayashida \& Akutsu, 2016). Dynamical gene expression data are also combined with network structure data in order to find pre-disease states $(\mathrm{Wu}$,
Chen, \& Wang, 2014). However, in these studies, the target types of cells or diseases are mostly limited to those related with specific diseases. Furthermore, most of these studies focus on practical aspects and thus it is not guaranteed to find the minimum set of marker genes. Therefore, in this paper, we consider the problem of identifying attractors by observing activities ( 0 or 1 ) of a small number of nodes using the BN model. In particular, we focus on finding the minimum number of nodes, by which all given attractors can be discriminated. We call this problem discrimination of attractors. Although it is computationally intractable (\#P-hard) to enumerate all singleton attractors (Akutsu, Kuhara, Maruyama, \& Miyano, 1998), some algorithms have been developed to enumerate all singleton attractors for up to moderate size networks by using network reduction (Veliz-Cuba, Aguilar, Hinkelmann, \& Laubenbacher, 2014) and to enumerate all singleton and periodic attractors by using network reduction and stable motifs (Zañudo \& Albert, 2013). Therefore, it is reasonable to assume that a set of attractors is given. Furthermore, we can even assume that this set is given independently of a $\mathrm{BN}$ because gene expression data for each cell type can be experimentally obtained without knowing the structure of the underlying genetic network.

In this paper, we begin with the discrimination problem for singleton attractors, and present an algorithm that works in polynomial time of the number of genes $(n)$ and the exponential factor only depends on the number of attractors $(m)$. Next, we present a key result, which states that any pair of (singleton and periodic) attractors can be discriminated by observing time-series data of two nodes, by making use of the Chinese Remainder Theorem. This result gives an upper bound of the number of sensor nodes to discriminate attractors. It also leads to development of an efficient algorithm whose polynomial degree depends only on the minimum number of sensor nodes. Then, we perform computational experiments using artificially generated BNs and BN models of real biological systems. Finally, we conclude with future work.

Note that Qiu et al. studied the same discrimination problem (Qiu, Cheng, Ching, Jiang, \& Akutsu, 2015). However, in their work, a very restricted model was considered: periodic attractors were not considered and discrimination nodes had to be selected from consecutive nodes. The latter restriction is too strong and is not appropriate from a biological viewpoint. Furthermore, such a restricted problem can be trivially solved in polynomial time because it is enough to examine all possible $O\left(n^{2}\right)$ intervals (although some improvement was done in their work). In this paper, we do not adopt such a non-realistic assumption. Cheng et al. also studied the same discrimination problem (Cheng, Qiu, Hou, \& Ching, 2017). They developed an integer programmingbased method for discrimination of singleton attractors. Although they also assumed that discrimination nodes had to be selected from consecutive nodes, this restric- 
tion can be easily removed and then the method might be practically useful. However, they did not perform any theoretical analysis and their method cannot handle periodic attractors. The main contributions of our work are discovery of a novel and useful property on periodic attractors, development of combinatorial algorithms for singleton and periodic attractors, and theoretical analyses of the time complexities of these algorithms. None of these was studied in the above-mentioned works.

\section{Discrimination of singleton attractors}

Before defining the problem, we briefly review Boolean networks (BNs). A BN $N(V, F)$ consists of a set of $n$ nodes $V=\left\{v_{1}, \ldots, v_{n}\right\}$ and a corresponding set of Boolean functions $F=\left\{f^{(i)} \mid v_{i} \in V\right\}$. Let $v_{i}(t) \in\{0,1\}$ represent the value of a node $v_{i}$ at time $t$, and denote by $\mathbf{v}(t)=\left(v_{1}(t), \ldots, v_{n}(t)\right)$ the state of the network at time $t$. The values of all nodes are updated simultaneously according to the corresponding Boolean functions, $v_{i}(t+1)=f^{(i)}(\mathbf{v}(t))$, where $f^{(i)}$ may depend only on a few nodes in $V$. A directed graph can be associated with the network in which there exists a directed edge $\left(v_{j}, v_{i}\right) \in E$ if and only if $f^{(i)}$ depends on $v_{j}$. Dynamics of a $\mathrm{BN}$ is well represented by a state transition diagram in which nodes correspond to network states and there exists a directed edge from $\mathbf{u}$ to $\mathbf{v}$ if and only if network state $\mathbf{u}$ at time $t$ transits to network state $\mathbf{v}$ at time $t+1$. A sequence of states $\mathbf{v}(0), \mathbf{v}(1), \ldots$, is called an attractor with period $p$ if $\mathbf{v}(0)=\mathbf{v}(p)$ and $\mathbf{v}(i) \neq \mathbf{v}(j)$ for all $i, j$ with $0 \leq i \neq j<p$. An attractor with period $p=1$ is called a singleton attractor. An attractor with $p>1$ is called a periodic attractor and is represented as $[\mathbf{v}(0), \mathbf{v}(1), \ldots, \mathbf{v}(p-1)]$. Note that if $[\mathbf{v}(0), \mathbf{v}(1), \ldots, \mathbf{v}(p-1)]$ is a periodic attractor, $\mathbf{v}(i)=\mathbf{v}(i+k p)$ holds for all $i, k$ with $i \geq 0$ and $k>0$. Therefore, $[\mathbf{v}(i), \mathbf{v}(i+1), \ldots, \mathbf{v}(i+p-1)]$ and $[\mathbf{v}(j), \mathbf{v}(j+1), \ldots, \mathbf{v}(j+p-1)]$ represent the same attractor for any $i, j$ with $i \neq j \geq 0$. We say that two periodic attractors $[\mathbf{v}(0), \mathbf{v}(1), \ldots, \mathbf{v}(p-1)]$ and $[\mathbf{u}(0), \mathbf{u}(1), \ldots, \mathbf{u}(p-1)]$ are identical if there exists an integer $k$ with $0<k<p$ such that $\mathbf{u}((i+k) \bmod p)=\mathbf{v}(i)$ holds for all $i=0,1, \ldots, p-1$ Fig. 1 shows an example of a BN. In this BN, transition rules are given by

$v_{1}(t+1)=v_{1}(t) \vee \overline{v_{3}(t)}$,

$v_{2}(t+1)=v_{2}(t) \wedge v_{3}(t)$

$v_{3}(t+1)=v_{1}(t) \oplus v_{2}(t)$,

where $x \wedge y, x \vee y, x \oplus y$, and $\bar{x}$ denote logical AND, OR, XOR, and NOT, respectively. There exist two singleton attractors, $(0,1,1)$ and $(1,0,1)$, and no periodic attractors.

Here we introduce the problem of determining the minimum discriminators for singleton attractors. Let $B$ be (a)

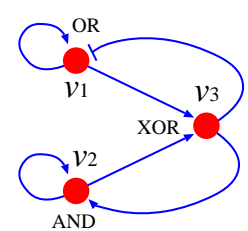

(b)

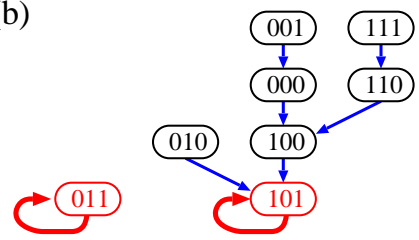

Fig. 1. Example of (a) BN and (b) its state transition diagram. T-type arrow in (a) means that the input is negated. In this BN, there exist two singleton attractors $(0,1,1)$ and $(1,0,1)$ which are shown in bold curves in (b).

an $m \times n$ binary matrix, where each row corresponds to a singleton attractor and each column corresponds to a node in a BN. $B[i, j]$ denotes the element at $i$-th row and $j$-th column. Here $B[i,-]$ and $B[-, j]$ denote the $i$-th row and $j$-th column of $B$, respectively. Let $J=\left\{j_{1}, \ldots, j_{k}\right\}$ be a set of column indices. Then, $B_{J}$ denotes the submatrix of $B$ consisting of the $j_{1}, j_{2}, \cdots, j_{k}$-th columns.

Definition 1 (Minimum Discriminator for Singleton Attractors [MinDiscSatt])

Input: $A$ set of singleton attractors represented as an $m \times n$ binary matrix $B$, where $m>1$ and $n>0$ correspond to the number of singleton attractors and the number of nodes (i.e., genes), respectively.

Output: $A$ minimum cardinality set $J$ of columns (i.e., nodes) such that $B_{J}\left[i_{1},-\right] \neq B_{J}\left[i_{2},-\right]$ holds for all $i_{1}, i_{2}$ with $1 \leq i_{1} \neq i_{2} \leq m$.

For example, consider the following matrix $B$ :

$$
\left(\begin{array}{llllll}
0 & 0 & 0 & 0 & 1 & 1 \\
1 & 1 & 1 & 1 & 0 & 0 \\
0 & 1 & 1 & 1 & 0 & 1 \\
0 & 0 & 1 & 0 & 1 & 1 \\
1 & 0 & 1 & 1 & 0 & 1 \\
1 & 0 & 1 & 1 & 0 & 0
\end{array}\right)
$$

Then, $J=\{2,3,5,6\}$ is a solution of MinDiscSatt for $B$ because any pair of rows are different in

$$
B_{\{2,3,5,6\}}=\left(\begin{array}{llll}
0 & 0 & 1 & 1 \\
1 & 1 & 0 & 0 \\
1 & 1 & 0 & 1 \\
0 & 1 & 1 & 1 \\
0 & 1 & 0 & 1 \\
0 & 1 & 0 & 0
\end{array}\right) .
$$

We remark that MinDiscSatt is a special case (i.e., binary case) of the minimum key problem (Akutsu \& Bao, 1996; Licchesi \& Osborn, 1978), and even the special case 
is known to be computationally intractable (NP-hard) (Motwani \& Xu, 2007).

The MinDiscSatt problem can be trivially solved in $O\left(2^{n} \operatorname{Poly}(m, n)\right)$ time by examining all possible $2^{n}$ subsets of columns, where Poly $(m, n)$ means some polynomial function of $m$ and $n$. However, $n$ is usually large and $m$ is usually small because $n$ corresponds to the number of genes and $m$ corresponds to the number of attractors (the number of different types of cells). In this case, $m^{m}<2^{n}$ may hold. In this paper, we present a simple dynamic programming algorithm that works in $O\left(m^{m} \operatorname{Poly}(m, n)\right)$ time.

Let $\mathbf{s}$ be an $m$-dimensional vector of integers between 0 to $m-1$, which we call a signature vector. Note that there are $m^{m}$ possible signature vectors. Here $\mathbf{0}$ denotes the signature vector consisting only of 0 's, and $\mathbf{s}_{i}$ denotes the $i$-th element of $\mathbf{s}$.

If each element of $\mathbf{s}$ is at most $2^{k}-1$ for some integer $k$ (i.e., each element of $\mathbf{s}$ is represented using $k$ bits), $\mathbf{s}$ is called a $k$-bit signature vector. Then, we identify such $\mathbf{s}$ with an $m \times k$ binary matrix by regarding the $i$-th element of $\mathbf{s}$ as the $i$-th row consisting of $k$ bits. Let $M(\mathbf{s})$ denote such a matrix. Conversely, we can construct a $k$-bit signature vector $\mathbf{s}$ from a given $m \times k$ binary matrix $M$ by identifying each row with a $k$ bit number. However, we use a compact form of $\mathbf{s}$ by renaming the numbers appearing in $\mathbf{s}$ (with the ordering being kept) so that only consecutive numbers beginning from 0 are used. Let the resulting vector be $\mathbf{v}(M)$. For an $m \times k_{1}$ matrix $M_{1}$ and $m \times k_{2}$ matrix $M_{2}, M_{1} \cdot M_{2}$ denotes $m \times\left(k_{1}+k_{2}\right)$ matrix obtained by concatenating $M_{1}$ and $M_{2}$. For example, consider the matrix $B$ given in the above. Then, for $\mathbf{s}=(1,6,6,3,2,2)$, $M(\mathbf{s})$ is a matrix consisting of the first three columns of $B_{\{2,3,5,6\}}$ and $M(\mathbf{s}) \cdot B[-, 6]=B_{\{2,3,5,6\}}$. While signature vectors corresponding to $B_{\{2,3,5\}}$ is $(1,6,6,3,2,2)$, $\mathbf{v}\left(B_{\{2,3,5\}}\right)=(0,3,3,2,1,1)$ (i.e., $1,6,3,2$ are replaced by $0,3,2,1$, respectively). Similarly, while signature vectors corresponding to $B_{\{2,3,5,6\}}$ is $(3,12,13,7,5,4)$, $\mathbf{v}\left(B_{\{2,3,5,6\}}\right)=(0,4,5,3,2,1)$. The reason why the compact form of a vector is employed is to use a dynamic programming table (explained below) of size $m^{m}(n+1)$.

We define a binary table $D[\mathbf{s}, k]$ by: $D[\mathbf{s}, k]=1$ if and only if there exists $J$ with $|J|=k$ such that $\mathbf{v}\left(B_{J}\right)=$ s. $D[\mathbf{s}, k]$ can be computed by the following dynamic programming procedure. Although it returns only the minimum size of $J$, such $J$ can be obtained by using the standard traceback procedure. All $D[\mathbf{s}, k] \mathrm{s}$ are initialized to be 0 .

$$
\begin{aligned}
& \text { Procedure SolveMinDiscSatt }(B) \\
& D[\mathbf{0}, 0] \leftarrow 1 ; \\
& \text { for } k=1 \text { to } n \text { do } \\
& \quad \text { for all } \mathbf{s} \text { such that } D[\mathbf{s}, k-1]=1 \text { do }
\end{aligned}
$$

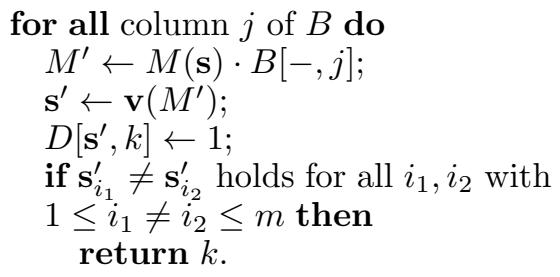

Since the total number of the compact forms of $\mathbf{s}$ should be smaller than or equal to $m^{m}$ and the other parts of SolveMinDiscSatt clearly work in $O(\operatorname{Poly}(m, n))$ time, we can see that MinDiscSatt can be solved by SolveMinDiscSatt in $O\left(m^{m} \operatorname{Poly}(m, n)\right)$ time.

This time complexity can be theoretically improved by identifying equivalent signatures (e.g., identifying $(0,1,2,2,3)$ and $(2,1,3,3,0))$. The result is summarized as below, where the algorithm, proof, and other details are given in Appendix A.

Theorem 1 MinDiscSatt can be solved in $O\left((\mathrm{~m} / 1.146)^{m}\right.$ Poly $(m, n))$ time.

\section{Discrimination of attractors}

In the above, we have considered discrimination of singleton attractors. Here, we consider the main problem: discrimination of periodic attractors, where singleton attractors can also be included. If we handle the discrimination problem as in MinDiscSatt, we may not give a correct solution. For example, suppose that two periodic attractors $A_{1}=[00,11]$ and $A_{2}=[01,10]$ are given. If we focus on 11 and 10, the solution of MinDiscSatt is $\left\{v_{2}\right\}$. However, the corresponding infinite time series data are $[0,1,0,1, \cdots]$ and $[1,0,1,0, \cdots]$ for $A_{1}$ and $A_{2}$, respectively. These two time series are identical by ignoring the starting states. Since we cannot know which is the starting state in practice, we cannot discriminate $A_{1}$ from $A_{2}$ by looking at time series data for $v_{2}$. The situation is the same for $v_{1}$. However, if we look at both $v_{1}$ and $v_{2}$, the time series are clearly different, $[00,11,00,11, \cdots]$ versus $[01,10,01,10, \cdots]$, and thus we can discriminate $A_{1}$ from $A_{2}$.

\subsection{Problem formulation}

For a set $U \subseteq V$ and an $n$-dimensional 0 -1 vector $\mathbf{v}$, $\mathbf{v}_{U}$ denotes the $|U|$-dimensional vector consisting of elements of $\mathbf{v}$ that correspond to $U$. For $v_{i} \in V, \mathbf{v}_{i}$ denotes the $i$-th coordinate value of $\mathbf{v}$. For example, if $n=5, \mathbf{v}=(1,0,1,1,0)$, and $U=\left\{v_{2}, v_{4}, v_{5}\right\}$, then $\mathbf{v}_{U}=(0,1,0)$ and $\mathbf{v}_{5}=0$. Let

$$
A_{h}=\left[\mathbf{v}(0), \mathbf{v}(1), \ldots, \mathbf{v}\left(p\left(A_{h}\right)-1\right)\right]
$$

be an attractor of period $p\left(A_{h}\right)$ (i.e., $\mathbf{v}(0)=\mathbf{v}\left(p\left(A_{h}\right)\right)$ ) on $N(V, F)$. Then, $\operatorname{Ser}\left(A_{h}, U, t\right)$ denotes an infinite se- 
quence of vectors defined by

$$
\operatorname{Ser}\left(A_{h}, U, t\right)=\left[\mathbf{v}_{U}(t), \mathbf{v}_{U}(t+1), \mathbf{v}_{U}(t+2), \cdots\right] .
$$

Note that two periodic attractors $A_{h}$ and $A_{k}$ are identical if and only if $\operatorname{Ser}\left(A_{h}, V, 0\right)=\operatorname{Ser}\left(A_{k}, V, t\right)$ holds for some $t \geq 0$. Then, our main problem is defined as below (see also Fig. 2).

\section{Definition 2 (Minimum Discriminator for Attractors} [MinDiscAtt])

Input: $A$ set of attractors $A=\left\{A_{1}, A_{2}, \ldots, A_{m}\right\}$, where each $A_{h}$ consists of $p\left(A_{h}\right)$ network states represented as a $p\left(A_{h}\right) \times n$ binary matrix, $p\left(A_{h}\right)$ denotes the period of $A_{h}, n>0$ denotes the number of nodes, and $m>1$. Output: A minimum cardinality set $U$ of nodes such that $\operatorname{Ser}\left(A_{h}, U, 0\right) \neq \operatorname{Ser}\left(A_{k}, U, t\right)$ for any $t \geq 0$ when $h \neq k$.

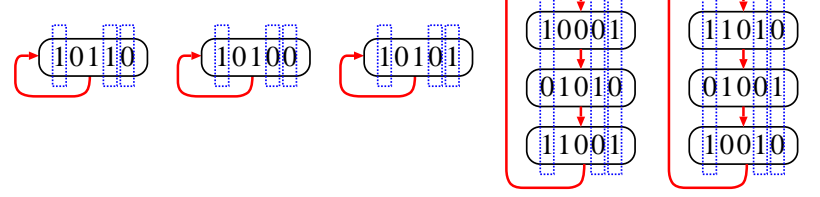

Fig. 2. Example of MinDiscAtt (Minimum Discriminator for Attractors). In this example, three singleton attractors and two periodic attractors are given as an input. The solution, $\left\{v_{1}, v_{4}, v_{5}\right\}$, is shown by dotted lines.

\subsection{Discrimination of two periodic attractors}

Before presenting an algorithm for MinDiscAtt, we show the key lemma which states that two periodic attractors can be discriminated by observing time series data of at most two nodes, by using the Chinese Remainder Theorem and its variant. Recall that the Chinese Remainder Theorem states that if $p_{1}, p_{2}, \ldots, p_{m}$ are pairwise coprime, for any integers $a_{1}, a_{2}, \ldots, a_{m}(0 \leq$ $\left.a_{i}<p_{i}\right)$, there exists a non-negative integer $x$ satisfying $x \equiv a_{i}\left(\bmod p_{i}\right)$ for all $i=1, \ldots, m$. Furthermore, the following variant is straight-forward from Theorem 10-3 and Theorem 10-4 of (Ore, 1988).

Proposition 1 If there exists a non-negative integer $x_{i j}$ satisfying $x_{i j} \equiv a_{i}\left(\bmod p_{i}\right)$ and $x_{i j} \equiv a_{j}\left(\bmod p_{j}\right)$ for each pair $(i, j)$ with $1 \leq i \neq j \leq m$, then there exists a non-negative integer $x$ satisfying $x \equiv a_{i}\left(\bmod p_{i}\right)$ for all $i$.

Lemma 1 For any two distinct (i.e., non-identical) attractors $A_{h}=\left[\mathbf{v}(0), \ldots, \mathbf{v}\left(p_{h}-1\right)\right]$ and $A_{k}=$ $\left[\mathbf{w}(0), \ldots, \mathbf{w}\left(p_{k}-1\right)\right]$, there exists $U \subseteq V$ of $|U|=2$ for which $\operatorname{Ser}\left(A_{h}, U, 0\right) \neq \operatorname{Ser}\left(A_{k}, U, t\right)$ holds for any $t \geq 0$.

Proof: If $p_{h}=1$ or $p_{k}=1$ holds, $A_{h}$ and $A_{k}$ can clearly be discriminated by looking at one node. Therefore, we assume $p_{h}>1$ and $p_{k}>1$.
For each node $v_{i}$ and a non-negative integer $t$, we define an infinite $0-1$ sequence $\operatorname{Dif}(i,-)$ by ${ }^{1}$

$\operatorname{Dif}(i, t)=\left\{\begin{array}{c}0, \text { if }\left[\mathbf{v}_{i}(0), \mathbf{v}_{i}(1), \mathbf{v}_{i}(2), \cdots\right]= \\ {\left[\mathbf{w}_{i}(t), \mathbf{w}_{i}(t+1), \mathbf{w}_{i}(t+2), \cdots\right]} \\ \text { holds, } \\ 1, \text { otherwise, }\end{array}\right.$

where $t \geq 0$. For a subset $U \subseteq V, \operatorname{Dif}(U,-)$ denotes a 0-1 table consisting of $\operatorname{Dif}(i,-)$ s for all $v_{i} \in U$, where each column corresponds to $v_{i}$ (see Table ??). Note that $\operatorname{Dif}(V, t)=00 \ldots 0$ holds for some $t$ if and only if $A_{h}$ and $A_{k}$ are identical.

We can see that for any fixed $i, \operatorname{Dif}(i, t)=1$ holds for all $t$, or $\operatorname{Dif}(i,-)$ has some period $p>1$ that is a divisor of the least common multiple of $p\left(A_{h}\right)$ and $p\left(A_{k}\right)$. In the latter case, 0 appears exactly once within each period $p$ (property (\#1)).

We can also see that $\operatorname{Dif}(U, t) \neq 00 \cdots 0$ holds for all $t$ if and only if $\operatorname{Ser}\left(A_{h}, U, 0\right) \neq \operatorname{Ser}\left(A_{k}, U, t\right)$ holds for all $t \geq 0$.

Here we note that since $A_{h}$ and $A_{k}$ are distinct periodic attractors, $\operatorname{Ser}\left(A_{h}, V, 0\right) \neq \operatorname{Ser}\left(A_{k}, V, t\right)$ holds for all $t \geq 0$, which also means that $\operatorname{Dif}(V, t) \neq 00 \cdots 0$ holds for all $t \geq 0$. If both $\operatorname{Dif}(i,-)$ and $\operatorname{Dif}(j,-)$ have the same period $p$ but $\operatorname{Dif}(i, t) \neq \operatorname{Dif}(j, t)$ holds for some $t, \operatorname{Dif}\left(\left\{v_{i}, v_{j}\right\}, t\right) \neq 00$ holds for all $t$ from (\#1). Then, we have a required $U$ (i.e., $U=\left\{v_{i}, v_{j}\right\}$ ).

Let $p_{i}$ be the period of $\operatorname{Dif}(i,-)$, and let $a_{i}$ be the smallest $t \geq 0$ such that $\operatorname{Dif}(i, t)=0$ (clearly, $a_{i}<p_{i}$ holds $)$.

If $p_{i} \mathbf{s}(i=1, \ldots, n)$ are pairwise coprime. There exists a non-negative integer $x$ such that $x \equiv a_{i}\left(\bmod p_{i}\right)$ from the Chinese Remainder Theorem. Then, $\operatorname{Dif}(V, x)=$ $00 \cdots 0$ must hold, which means that $A_{h}$ and $A_{k}$ are identical periodic attractors. This contradicts with that $A_{h}$ and $A_{k}$ are non-identical.

If $p_{i}$ s are not pairwise coprime (i.e., there exists some non-coprime pair $\left.\left(p_{i}, p_{j}\right)\right)$. Suppose that $A_{h}$ and $A_{k}$ are not identical but, for any $U=\left\{v_{i}, v_{j}\right\} \subseteq V$, $\operatorname{Dif}\left(U, t_{i j}\right)=00$ holds for some $t_{i j}$ (it means $\bar{t}_{i j} \equiv$ $a_{i}\left(\bmod p_{i}\right)$ and $\left.t_{i j} \equiv a_{j}\left(\bmod p_{j}\right)\right)$. We can see from Prop. 1 that there exists a non-negative integer $x$ satisfying $x \equiv a_{i}\left(\bmod p_{i}\right)$ for all $i=1, \ldots, n$, from which $\operatorname{Dif}(V, x)=00 \cdots 0$ follows. It contradicts the assumption that $A_{h}$ and $A_{k}$ are not identical. Therefore, if $A_{h}$

${ }^{1}$ We use $\operatorname{Dif}(i,-)$ to denote a sequence for $t \geq 0$ and $\operatorname{Dif}(i, t)$ to denote a specific item at time $t$ in the sequence. 
and $A_{k}$ are not identical, there exists $U$ of $|U|=2$ such that $\operatorname{Dif}(U, t) \neq 00$ holds for all $t$.

Lemma 1 indicates that the minimum number of nodes for discriminating two different periodic attractors is at most two. Therefore, we can see that the size of $U$ in MinDiscAtt is upper-bounded by $m \cdot(m-1)$.

\subsection{Discrimination of $m$ attractors}

Here we present an algorithm to select a minimum set of nodes for discriminating given $m$ attractors, which are singleton or periodic. Let $A=\left\{A_{1}, \ldots, A_{m}\right\}$ be a set of singleton and periodic attractors. We use $\operatorname{Dif}_{(h, k)}(i, t)$ to denote $\operatorname{Dif}(i, t)$ for a pair $\left(A_{h}, A_{k}\right)$. We employ two matrices $P_{A}$ and $R_{A}$ to record the information of these $m$ attractors. The sizes of $P_{A}$ and $R_{A}$ are the same, which equals to $(m(m-1) / 2) \times n$. The rows of $P_{A}$ and $R_{A}$ are indexed by the pairs $G=\{(h, k) \mid 0 \leq h<k \leq m\}$. In order to explicitly represent the index, we employ a function $g$ that gives a one-to-one mapping from a set of $(h, k)$ s with $1 \leq h<k \leq m$ to a set of integers between 1 and $m(m-1) / 2$. For example, we can use $g$ defined by

$$
\begin{aligned}
g(h, k) & =\left\{\sum_{t=1}^{h-1}(m-t)\right\}+(k-h) \\
& =(h-1) m-\frac{h(h-1)}{2}+(k-h) .
\end{aligned}
$$

The elements of $P_{A}[g(h, k), i]$ and $R_{A}[g(h, k), i]$ are defined by

- If $\operatorname{Ser}\left(A_{h}, i, 0\right)=\operatorname{Ser}\left(A_{k}, i, t\right)$ for all $t$, (it means that $\operatorname{Ser}\left(A_{h}, i, 0\right)$ and $\operatorname{Ser}\left(A_{k}, i, 0\right)$ are infinite sequences of the same number $(0$ or 1$)), P_{A}[g(h, k), i]=1$ and $R_{A}[g(h, k), i]=0$.

- If $\operatorname{Ser}\left(A_{h}, i, 0\right)=\operatorname{Ser}\left(A_{k}, i, t\right)$ not for all $t$ but for some $t, P_{A}[g(h, k), i]$ is the period of $\operatorname{Dif}_{(h, k)}(i,-)$ of these two attractors and $R_{A}[g(h, k), i]$ equals to the position of $0\left(\bmod P_{A}[g(h, k), i]\right)$.

- Otherwise (i.e., $\operatorname{Ser}\left(A_{h}, i, 0\right) \neq \operatorname{Ser}\left(A_{k}, i, t\right)$ for all $\left.t\right)$, $P_{A}[g(h, k), i]=R_{A}[g(h, k), i]=0$.

For MinDiscAtt, we should find a minimum set $J$ such that for all $g=g(h, k)$, either there exists 0 in $P_{A}[g, j]$ for some $j \in J$, or, $R_{A}[g, i] \neq$ $R_{A}[g, j]\left(\bmod \operatorname{gcd}\left(P_{A}[g, i], P_{A}[g, j]\right)\right)$ holds for some $i, j \in J$ with $i \neq j$.

Example 1 Suppose $\left\{A_{1}, A_{2}, A_{3}\right\}$ are given in Table??, then $P_{A}$ and $R_{A}$ are given as follows.

$$
P_{A}=\left[\begin{array}{llll}
6 & 3 & 2 & 2 \\
0 & 0 & 2 & 0 \\
0 & 0 & 2 & 0
\end{array}\right] \quad R_{A}=\left[\begin{array}{llll}
0 & 0 & 1 & 0 \\
0 & 0 & 0 & 0 \\
0 & 0 & 1 & 0
\end{array}\right]
$$

Now, we have the algorithm SolveMinDiscAtt as follows.

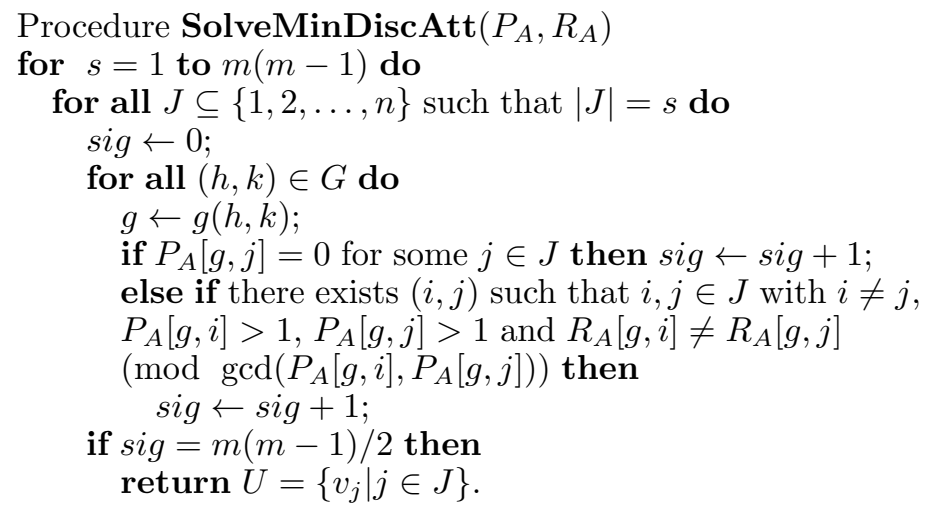

Here is an example to illustrate the above algorithm.

Example 2 Suppose that $P_{A}$ and $R_{A}$ are the same as in Example 1. First, we consider the case of $s=1$. Since $|J|=1$, the condition of else if part is trivially not satisfied. Since there is no column consisting of only 0s, any $J$ of $|J|=1$ cannot satisfy the condition of if part in for loop for $m(m-1) / 2$ times. Therefore, any $J$ with $|J|=1$ is not a solution. Next, SolveMinDiscAtt examines $J$ with $|J|=2$. For each $J$, it checks whether one of the following conditions is satisfied for each $g=g(h, k)$ (i.e., a pair of attractors), where each row of $P_{A}$ (resp., $R_{A}$ ) corresponds to a pair of attractors.

(1) There exists $j \in J$ such that $P_{A}[g, j]=0$.

(2) There exists $(i, j)$ such that $i \neq j \in J, P_{A}[g, i]>1$, $P_{A}[g, j]>1$, and $R_{A}[g, i] \neq R_{A}[g, j]\left(\bmod g c d\left(P_{A}[g, i]\right.\right.$, $\left.\left.P_{A}[g, j]\right)\right)$.

Let sig represent the total number of pairs of attractors satisfying one of these two conditions. Initially, sig is set as 0. Suppose that we consider the relation between $A_{1}$ and $A_{2}$, which is represented by the first row of $P_{A}$. When $J=\{1,2\}$, we have $R_{A}(1,1)=$ $R_{A}(1,2)\left(\bmod \operatorname{gcd}\left(P_{A}(1,1), P_{A}(1,2)\right)\right)$. Therefore, $J=\{1,2\}$ cannot discriminate $A_{1}$ and $A_{2}$. Similar results can be obtained for $J=\{1,4\},\{2,4\},\{2,3\}$. As for $J=\{3,4\}$, we can check that $R_{A}[1,3] \neq$ $R_{A}[1,4]\left(\bmod \operatorname{gcd}\left(P_{A}[1,3], P_{A}[1,4]\right)\right)$. Furthermore, for the second and third rows, we have $P_{A}[2,4]=P_{A}[3,4]=$ 0 . Since $m(m-1) / 2=3$ for $m=3$ and sig reaches 3 , these 3 periodic attractors can be distinguished from each other by observing $v_{3}$ and $v_{4}$.

By using SolveMinDiscAtt, we have the following theorem where the proof is given in Appendix B.

Theorem 2 MinDiscAtt can be solved in $O\left(n^{\left|U_{\min }\right|}\right.$ Poly $(m, n, p))$ time, where $U_{\min }$ is an optimal solution 
of MinDiscAtt and $p$ is the longest period of input attractors. Furthermore, $\left|U_{\min }\right| \leq m(m-1)$ holds.

\subsection{Remark on feedback vertex set}

Akutsu et al. introduced the Feedback Vertex Set (FVS) to BNs for efficiently identifying singleton attractors (Akutsu et al., 1998). FVS is a well-known concept in graph theory and is a set of nodes whose removal makes a graph acyclic. Mochizuki et al. showed that all singleton and periodic attractors can be identified by observing states of nodes in an FVS (Mochizuki, Fiedler, Kurosawa, \& Saito, 2013). This fact implies that an FVS gives a discriminator set for singleton and periodic attractors only from the network structure, independently of Boolean functions assigned to nodes. Moreover, Aracena showed that if a BN is a network with signs on arcs, a minimum positive feedback vertex set (a minimum set of nodes whose removal leaves a digraph without positive cycles) discriminates singleton attractors, which is often smaller than a minimum FVS (Aracena, 2008). Here we give a very simple example showing that the minimum FVS does not necessarily give an optimal solution of MinDiscSatt or MinDiscAtt (i.e., a minimum discriminator), depending on Boolean functions assigned to nodes. Consider a BN shown in Fig. 1. In this $\mathrm{BN}$, there exist two singleton attractors: $(0,1,1)$ and $(1,0,1)$. These two attractors can be discriminated by observing the state of $v_{1}$ (or $v_{2}$ ). Therefore, the size of the minimum discriminator node set is 1 . On the other hand, the minimum FVS is $\left\{v_{1}, v_{2}\right\}$. Therefore, we can see that the minimum FVS does not necessarily give an optimal solution of MinDiscSatt or MinDiscAtt. It is interesting to note that a similar observation was done on a kind of control problem on attractors (Zañudo \& Albert, 2015).

Of course, if other Boolean functions are assigned even with keeping the network structure, the situation changes. For example, consider the following BN:

$v_{1}(t+1)=v_{1}(t) \vee \overline{v_{3}(t)}$,

$v_{2}(t+1)=v_{2}(t) \wedge v_{3}(t)$

$v_{3}(t+1)=v_{1}(t) \vee v_{2}(t)$.

Then, the singleton attractors of this $\mathrm{BN}$ are $(1,1,1)$, $(0,1,1)$, and $(1,0,1)$. In this case, the minimum FVS $\left\{v_{1}, v_{2}\right\}$, which is also the minimum positive FVS, gives an optimal solution of MinDiscSatt. These examples imply that the minimum discriminator set depends not only on the network structure but also on Boolean functions assigned to nodes of a BN. Although self loops are included in this BN, we can construct a BN without selfloop. For example, consider the BN defined by

$$
\begin{aligned}
& v_{1}(t+1)=v_{2}(t) \wedge v_{3}(t) \\
& v_{2}(t+1)=v_{1}(t) \wedge v_{3}(t) \\
& v_{3}(t+1)=v_{1}(t) \wedge v_{2}(t)
\end{aligned}
$$

Then, there exist two singleton attractors $((0,0,0)$ and $(1,1,1))$ and no periodic attractor, whereas the size of the minimum FVS is 2 . Of course, all the theoretical results in this paper hold regardless of existence of selfloops.

\section{Results of computational experiments}

In order to evaluate our developed algorithms, we conducted numerical experiments with Intel FORTRAN on a PC with Intel dual-core $2.6 \mathrm{GHz}$ processor and $4 \mathrm{G}$ RAM, where SolveMinDiscAtt was slightly modified to improve the practical efficiency. The source and executable codes of the developed programs are available from http://hkumath.hku.hk/ ${ }^{\sim}$ wkc/Cheng/Discrimination-of-Attractors.zip .

\subsection{Results on artificial data}

We tested the efficiency of our proposed algorithms using randomly generated attractors. Results by SolveMinDiscSatt and SolveMinDiscAtt are shown in Tables 3 and ??, respectively.

We have generated a couple of $m \times n$ matrices, where $m$ is the number of singleton attractors and $n$ is the number of nodes. It is seen from Table 3 that the minimum number of nodes to discriminate $m$ attractors is around $\lceil\log (m)]$, which is reasonable since the binary matrices are randomly generated.

For each experiment of SolveMinDiscAtt, there exist 5 periodic attractors, whose lengths are bounded by 10 . Each case is represented by a $1 \times 5$ vector $\mathbf{p}$ in which each element represents the length of a periodic attractor. For a fixed p, we randomly generated 10 sets of periodic attractors (same length but different matrices). The number of nodes was set as 100 or 1000 . For each case, we computed the average over 10 trials. The results are shown in Table ??. The size of each solution was 1 , which is much smaller than the theoretically derived upper bound, $m(m-1)$. It is not surprising that 5 periodic attractors can be discriminated by looking at only one node because it is expected that randomly generated 0-1 sequences are pairwise non-identical even for one node.

Table 3

Time cost of SolveMinDiscSatt applied to randomly generated attractors.

\begin{tabular}{cccc}
\hline$m$ & $n$ & Time(s) & Min Node \\
\hline 5 & 100 & $<10^{-5}$ & 3 \\
5 & 10000 & $<10^{-5}$ & 3 \\
7 & 10000 & 0.1875 & 3 \\
10 & 10000 & 15294 & 4 \\
\hline
\end{tabular}




\subsection{Results on biological data}

We also applied SolveMinDiscSatt and SolveMinDiscAtt to four biological processes: the expression pattern of the segment polarity genes in Drosophila Melanogaster (Albert \& Othmer, 2003), which includes 60 genes (15 genes each for four different cells) and 10 singleton attractors, the control of the mammalian cell cycle (Fauré et al., 2006), which includes 10 genes, one singleton attractor and one periodic attractor of length 7, the logical model analyzing T-cell activation (Klamt, Saze-Rodriguez, Lindquist, Simeoni, \& Gilles, 2006), which includes 40 genes, 8 singleton attractors and one periodic attractor of length 6 , and a Boolean model of IGVH mutational status in chronic lymphocytic leukemia (Alvarez-Silva, Yepes, Torres, \& Barrios, 2015), which includes 90 genes, 6 periodic attractors all with period 4 .

We have applied SolveMinDiscSatt to the Drosophila Melanogaster model and SolveMinDiscAtt to the other three processes. Recall that SolveMinDiscAtt can also handle singleton attractors. The results are shown in Table ??. In the table, "Time" means the time needed for finding the minimum set of nodes, "Min Node" means the size of the minimum set of nodes, and "Genes" means gene names of these nodes. As for the first process, we do not record the minimum set of nodes but only maintain its size via $D[\mathbf{s}, k]$ when applying SolveMinDiscSatt, but the set can be obtained by tracing back.

It seems that biologically important genes were identified for these network models. Here, we briefly discuss the roles of identified genes. The $w g$ genes activate WG (wingless genes), and WG genes regulate en genes. Note that in Table ??, subscripts 1 and 2 correspond to the first and second cells, respectively (e.g., $\mathrm{wg}_{1}$ denotes the wg gene in the first cell). The engrailed (en) mutation leads to the transformation of the posterior structures of the dorsal mesothoracic disc into those characteristic of the anterior region of the same disc (GarcíaBellido \& Santamaría, 1972). PTC (Patched) regulates itself and SMO. SMO (Smoothened) is segment polarity gene required for correct patterning of every segment in Drosophila (Alcedo, Ayzenzon, Von Ohlen, Noll, \& Hooper, 1996).

Mammalian cell division starts after $\mathrm{CycD}$ (Cyclin D) is activated by positive signals or growth factor. $\mathrm{CycD}$ is not active in the stable state, but active in the dynamic cycle for the synchronous model (Fauré et al., 2006).

CD45 is a leucocyte common antigen glycoprotein, which binds with T-cell receptor (TCR) and activates Fyn. CD8 is known to be active in cytotoxic T-cells. TRCbind represents whether TCR is bound with antigen (Klamt, Saez-Rodriguez, \& Gilles, 2007).
AEBP1 (AE binding protein) plays a key role in modulation of adiposity via fat-cell proliferation (Zhang, Reidy, Nicholson, Lee, Majdalawieh, Webber, Stewart et al., 2005). CCND2 (Cyclin D2) is implicated in cell cycle regulation, differentiation, and oncogenic transformation (Meyyappan, Wong, Hull, \& Riabowol, 1998). INPP5D (Inositol Polyphosphate-5-Phosphatase D) regulates diverse cellular processes such as protein trafficking, phagocytosis and synaptic vesicle recycling (Ooms, Horan, Rahman, Seaton, Gurung, Kethesparan et al., 2009).

\section{Conclusion}

Observability of complex networks plays an important role in diagnosing complex systems, especially for complex biological systems. If we need to know the states of the whole system, a lot of nodes should be selected as sensor nodes, which is not realistic. Therefore, we have introduced the concept of discrimination of attractors in this paper, which might be useful to identify cell types. We defined MinDiscSatt and MinDiscAtt for selecting the minimum set of sensor nodes to discriminate singleton and periodic/singleton attractors, respectively, and developed corresponding algorithms.

The results of computational experiments on artificially generated data and biological network data suggest that we need a small number of sensor nodes in practice, especially for discrimination of periodic attractors. This observation might lead to development of efficient diagnosis methods of cell types and/or biological networks. Of course, in order to handle real data such as gene expression data, Boolean modeling is not enough. Real data contain noises and are usually given as real valued ones. Therefore, further studies should be done to extend the methodologies introduced in this paper to continuous models and stochastic models.

\section{Appendix A. Improved algorithm for discrimina- tion of singleton attractors}

This section presents an improved algorithm for discrimination of singleton attractors. As mentioned in the main text, the key idea is to identify equivalent signatures (e.g., $(0,1,2,2,3)$ and $(2,1,3,3,0))$, where two signatures are called equivalent if they have the same canonical form (which is defined below). In order to analyze the time complexity while identifying equivalent signatures, we only consider vectors in the canonical form. For a vector $\mathbf{s}$, let $N(\mathbf{s})$ denotes the set of integer numbers appearing in $\mathbf{s}$. For example, $N(\mathbf{s})=\{0,1,2,3\}$ for $\mathbf{s}=(2,1,3,3,0)$. We consider a one-to-one mapping $\phi$ from $N(\mathbf{s})$ to the set of integers between 0 and $|N(\mathbf{s})|-1$ and let $\phi(\mathbf{s})$ be the vector obtained by replacing the $i$ th element $\mathbf{s}_{i}$ of $\mathbf{s}$ with $\phi\left(\mathbf{s}_{i}\right)$ for all $i$. Then, $\phi(\mathbf{s})$ is called the canonical form if the first occurrence 
of an integer $i$ in $\phi(\mathbf{s})$ precedes the first occurrence of an integer $j$ for any $j>i$. It is obvious that the canonical form is uniquely determined for each vector $\mathbf{s}$. We use $\alpha(\mathbf{s})$ to denote the canonical form of $\mathbf{s}$. For example. $\alpha\left(\mathbf{s}^{1}\right)=(0,1,2,2,3,0,0)$ for $\mathbf{s}^{1}=(2,1,3,3,0,2,2)$ and $\alpha\left(\mathbf{s}^{2}\right)=(0,1,1,2,3,2,2)$ for $\mathbf{s}^{2}=(5,2,2,7,1,7,7)$.

By using this canonical form, we obtain an improved algorithm ImpSolveMinDiscSatt as below, where we consider table entries $D[\mathbf{s},-]$ only for canonical vectors s. Note that ImpSolveMinDiscSatt differs from SolveMinDiscSatt only in that $\alpha\left(\mathbf{v}\left(M^{\prime}\right)\right)$ is used instead of $\mathbf{v}\left(M^{\prime}\right)$.

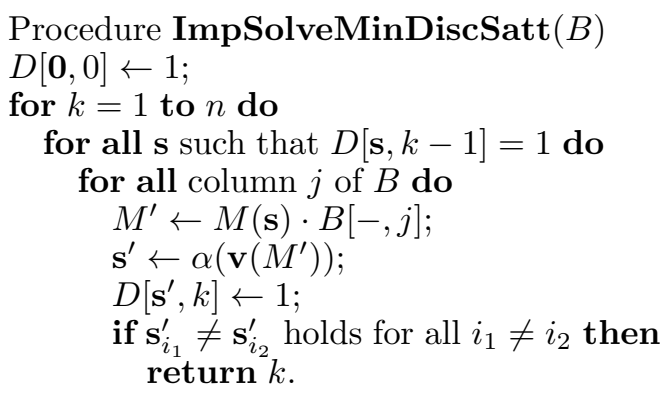

Proof of Theorem 1: Since the correctness of ImpSolveMinDiscSatt is obvious, we consider the time complexity.

First of all, we analyze the number of canonical forms for vectors of length $m$. If an integer $i$ appears multiple times (more than one time) in a vector $\mathbf{s}, i$ is called a repeater. We calculate the total number of canonical forms having $k$ repeaters. For example, $\mathbf{s}=(0,1,2,3,1,4,3,1)$ has two repeaters: 1 and 3 . Let $f(k, m)$ denote the total number of canonical vectors of length $m$ having $k$ repeaters.

If $k=0$, all the elements of $\mathbf{s}$ are different. Since such a canonical vector is unique (i.e., $\mathbf{s}=(0,1,2, \ldots, m))$, $f(0, m)=1$. If $k=1$, there exists only one repeater. Let $j$ denote the number of occurrences of this repeater. Then $j \in\{2,3, \ldots, m\}$, and all other elements are different and are determined uniquely. Therefore, $f(1, m)=$ $\sum_{i=2}^{m}\left(\begin{array}{c}m \\ i\end{array}\right)$.

For a general $k$, we have

$f(k, m) \leq \sum_{i=2}^{m-2(k-1)}\left(\begin{array}{c}m \\ i\end{array}\right) f(k-1, m-i)$.

For a fixed $m$, let $F(m)$ denote the total number of canonical vectors of length $m$. Then, we have

$$
\begin{aligned}
F(m) & =\sum_{k=0}^{\lfloor m / 2\rfloor} f(k, m) \\
& \leq 1+\sum_{k=1}^{\lfloor m / 2\rfloor} \sum_{i=2}^{m-2(k-1)}\left(\begin{array}{c}
m \\
i
\end{array}\right) f(k-1, m-i) \\
& =1+\sum_{i=2}^{m} \sum_{k=1}^{\lfloor(m-i+2) / 2\rfloor}\left(\begin{array}{c}
m \\
i
\end{array}\right) f(k-1, m-i) \\
& =1+\sum_{i=2}^{m}\left(\begin{array}{c}
m \\
i
\end{array}\right) \sum_{k=0}^{\lfloor(m-i) / 2\rfloor} f(k, m-i) \\
& =1+\sum_{j=0}^{m-2}\left(\begin{array}{c}
m \\
j
\end{array}\right) F(j)
\end{aligned}
$$

Here $F(0)=F(1)=1, F(2)=2$, and $F(3)=5$. By mathematical induction, we will prove $F(m)<(m / a)^{m}$ for all $m \geq 2$, where $a$ is a constant to be determined as below. For a while, we assume $a<1.15$. Clearly, it holds for $m=2$ and $m=3$. Suppose that $F(i)<(i / a)^{i}$ holds for $2 \leq i<m-1$. Then,

$$
\begin{aligned}
F(m) & \leq 1+\sum_{j=0}^{m-2}\left(\begin{array}{c}
m \\
j
\end{array}\right) F(j) \\
& <1+F(0)+m \cdot F(1)+\sum_{j=2}^{m-2}\left(\begin{array}{c}
m \\
j
\end{array}\right)(j / a)^{j} \\
& <\sum_{j=0}^{m-2}\left(\begin{array}{c}
m \\
j
\end{array}\right)(m / a)^{j} \\
& =(m / a+1)^{m}-(m / a)^{m}-(m / a)^{m-1} \cdot m
\end{aligned}
$$

holds for $m \geq 4$. Now, we have to prove

$$
(m / a+1)^{m}-(m / a)^{m}-(m / a)^{m-1} \cdot m<(m / a)^{m},
$$

which is equivalent to

$$
(m / a+1)^{m}<2(m / a)^{m}+(m / a)^{m-1} \cdot m .
$$

By dividing both sides by $(m / a)^{m}$, we obtain

$$
\left\{(1+a / m)^{m / a}\right\}^{a}<2+a .
$$

Since $\left\{(1+a / m)^{m / a}\right\}^{a}<e^{a}$, it is enough to guarantee $e^{a}<2+a$. By numerical calculation, we can see that $e^{a}<2+a$ holds for $a \leq 1.146$.

Since $O(\operatorname{Poly}(m, n))$ time is required per canonical vector in ImpSolveMinDiscSatt, the total time complexity is $O\left((m / 1.146)^{m} \operatorname{Poly}(m, n)\right)$.

Although this result improves the theoretical time complexity from $O\left(m^{m} \operatorname{Poly}(m, n)\right)$ to $O\left((m / 1.140)^{m} \operatorname{Poly}(m, n)\right)$, there is $O(m)$ additional factor hidden in the $\operatorname{Poly}(m, n)$ 
notation for computing $\alpha\left(\mathbf{v}\left(M^{\prime}\right)\right)$ (e.g., by radix sort), Therefore, ImpSolveMinDiscSatt is better than SolveMinDiscSatt only if $(1,146)^{m} \geq m$ holds (ignoring a constant factor). Since $(1,146)^{m} \geq m$ is satisfied only for $m>23$, ImpSolveMinDiscSatt is not better than SolveMinDiscSatt and the time complexity of ImpSolveMinDiscSatt is too high for $m>23$, ImpSolveMinDiscSatt is not practical and is only of theoretical interest.

\section{Appendix B. Proof of Theorem 2}

First we show the correctness of the algorithm. From Lemma 1 , it is seen that for each pair $(h, k)$ with $1 \leq h \neq k \leq m$, there exists $\left\{v_{i}, v_{j}\right\} \subseteq V$ such that $\operatorname{Ser}\left(A_{h},\left\{v_{i}, \bar{v}_{j}\right\}, 0\right) \neq \operatorname{Ser}\left(A_{k},\left\{v_{i}, v_{j}\right\}, \bar{t}\right)$ holds for all $t \geq 0$.

As a special case, suppose that $\operatorname{Ser}\left(A_{h},\left\{v_{i}\right\}, 0\right) \neq$ $\operatorname{Ser}\left(A_{k},\left\{v_{i}\right\}, t\right)$ holds for all $t \geq 0$. From the definition of $P_{A}[g(h, k), i]$, it is equivalent to $P_{A}[g(h, k), i]=0$. Furthermore, it corresponds to the case that $P_{A}[g(h, k), j]=$ 0 holds for some $j \in J$. Note that this case includes the case of $p\left(A_{h}\right)=1$ or $p\left(A_{k}\right)=1$.

Next, we consider the case that $\operatorname{Ser}\left(A_{h},\left\{v_{i}, v_{j}\right\}, 0\right) \neq$ $\operatorname{Ser}\left(A_{k},\left\{v_{i}, v_{j}\right\}, t\right)$ holds for all $t$, which is equivalent to $D i f_{(h, k)}\left(\left\{v_{i}, v_{j}\right\}, t\right) \neq 00$ for all $t$. Recall that $R_{A}[g, i]=$ $a_{i}$ means that $a_{i}$ is the minimum number such that $\operatorname{Dif}_{(h, k)}\left(i, a_{i}\right)=0$ holds. Thus, Dif $f_{(h, k)}(i, t)=0$ holds if and only if $t=a_{i}+n_{i} p_{i}$ for some integer $n_{i} \geq 0$. Therefore, $\operatorname{Dif}_{(h, k)}\left(\left\{v_{i}, v_{j}\right\}, t\right)=00$ holds if and only if $a_{i}+n_{i} p_{i}=a_{j}+n_{j} p_{j}=t$. Let $d=\operatorname{gcd}\left(p_{i}, p_{j}\right)$. We assume without loss of generality that $a_{i} \geq a_{j}$ holds. Then, we have

$$
\begin{aligned}
a_{i}+n_{i} p_{i} & =a_{j}+n_{j} p_{j}, \\
a_{i}+n_{i} q_{i} d & =a_{j}+n_{j} q_{j} d, \\
a_{i}-a_{j} & =\left(n_{j} q_{j}-n_{i} q_{i}\right) d .
\end{aligned}
$$

The last equality is equivalent to $a_{i} \equiv a_{j}\left(\bmod \operatorname{gcd}\left(p_{i}, p_{j}\right)\right)$. Therefore, the algorithm correctly identifies the minimum $U$.

Finally, we consider the time complexity. Clearly, the algorithm examines $O\left(n^{\left|U_{\min }\right|}\right)$ combinations of $J$. Since the other parts of SolveMinDiscAtt work in $O(\operatorname{Poly}(m, n, p))$ time per $J$, the total time complexity is $O\left(n^{\left|U_{\min }\right|} \operatorname{Poly}(m, n, p)\right)$. Furthermore, since two nodes are enough to discriminate two attractors and there are $m(m-1) / 2$ pairs of attractors, $\left|U_{\min }\right| \leq m(m-1)$ holds.

\section{References}

Akutsu, T., \& Bao, F. (1996). Approximating minimum keys and optimal substructure screens. In Proceedings of
Second Annual International Conference on Computing and Combinatorics (pp. 290-299). Berlin: Springer.

Akutsu, T., Kuhara, A., Maruyama, O., \& Miyano, S. (1998). A system for identifying genetic networks from gene expression patterns produced by gene disruptions and overexpressions. Genome Informatics, 9, 151-160.

Albert, R., \& Othmer, H. (2003). The topology of the regulatory interactions predicts the expression pattern of the segment polarity genes in Drosophila Melanogaster. Journal of Theoretical Biology, 223(1), 911-918.

Albert, R., \& Thakar, J. (2014). Boolean modeling: a logicbased dynamic approach for understanding signaling and regulatory networks and for making useful predictions. WIREs Systems Biology and Medicine, 6(5), 353-369.

Alcedo, J., Ayzenzon, M., Von Ohlen, T., Noll, M., \& Hooper, J. E. (1996). The Drosophila smoothened gene encodes a sevenpass membrane protein, a putative receptor for the hedgehog signal. Cell, 86(2), 221-232,

Alvarez-Silva, M., Yepes, A., Torres, M., \& Barrios, A. (2015). Protein interaction network and modeling of IGVH mutational status in chronic lymphocytic leukemia. Theoretical Biology and Medical Modelling, 12, 12.

Aracena, J. (2008). Maximum number of fixed points in regulatory Boolean networks. Bulletin of Mathematical Biology, 70, 1398-1409.

Cheng, D., Qi, H., \& Li, Z. (2011). Analysis and Control of Boolean Networks. Berlin: Springer.

Cheng, X., Qiu, Y., Hou, W., \& Ching, W-K. (2017). Integer programming-based method for observability of singleton attractors in Boolean networks. IET Systems Biology, 11, 3035

Drossel, B., Mihaljev, T., \& Greil, F. (2005).

Number and length of attractors in a critical Kauffman model with connectivity one. Physical Review Letters, 94, 088701.

Fauré, A., Naldi, A., Chaouiya, C., \& Thieffry, D. (2006). Dynamical analysis of a generic Boolean model for the control of the mammalian cell cycles. Bioinformatics, 22(14), e124e131.

García-Bellido, A., \& Santamaría, P. (1972). Developmental analysis of the wing disc in the mutant engrailed of Drosophila melanogaster. Genetics, 72(1), 87-104.

Garg, A., Di Cara, A., Xenarios, I., Mendoza, L., \& De Micheli, G. (2008). Synchronous versus asynchronous modeling of gene regulatory networks. Bioinformatics, 24(17), 1917-1925.

Harvey, I., \& Bossomaier, T. (1997).

Time out of joint: Attractors in asynchronous random Boolean networks. In Proceedings of the Fourth European Conference on Artificial Life (pp. 67-75). Cambridge MA: MIT Press

Hayashida, M., \& Akutsu, T. (2016). Complex network-based approaches to biomarker discovery. Biomarkers in Medicine, 10(6), 621-632.

Kauffman, S. (1993). The Origins of Order: Self-organization and Selection in Evolution. Oxford UK: Oxford Univ. Press.

Kauffman, S., Peterson, C., Samuelsson, B., \& Troein, C. (2004). Genetic networks with canalyzing Boolean rules are always stable. Proceedings of National Academy of Sciences, USA, 101(49), 17102-17107.

Klamt, S., Saez-Rodriguez, J., \& Gilles, E. (2007). Structural and functional analysis of cellular networks with CellNetAnalyzer. BMC Systems Biology, 1(1), 2.

Klamt, S., Saze-Rodriguez, J., Lindquist, J., Simeoni, L., \& Gilles, E. (2006). A methodology for the structural and 
functional analysis of signaling and regulatory networks. $B M C$ Bioinformatics, 7, 56.

Laschov, D., Margaliot, M., \& Even, G. (2013). Observability of Boolean networks: A graph-theoretic approach. Automatica, 49(8), 2351-2362.

Li, R., Yang, M., \& Chu, T. (2015).

Controllability and observability of Boolean networks arising from biology. Chaos, 24, 023104.

Licchesi, C., \& Osborn, S. (1978). Candidate keys for relations. Journal of Computer and System Sciences, 17(2), 270-279.

Liu, Y., Slotine, J., \& Barabàsi, A. (2013).

Observability of complex systems. Proceedings of National Academy of Sciences, USA, 110(7), 2460-2465.

Meyyappan, M., Wong, H., Hull, C., \& Riabowol, K. T. (1998). Increased expression of cyclin D2 during multiple states of growth arrest in primary and established cells. Molecular and Cellular Biology, 18(6), 3163-3172.

Mochizuki, A. (2005). An analytical study of the number of steady states in gene regulatory networks. Journal of Theoretical Biology, 236, 291-310.

Mochizuki, A., Fiedler, B., Kurosawa, G., \& Saito, D. (2013). Dynamics and control at feedback vertex sets. II: A faithful monitor to determine the diversity of molecular activities in regulatory networks. Journal of Theoretical Biology, 335, 130146.

Motwani, R., \& Xu, Y. (2007). Efficient algorithms for masking and finding quasi-identifiers, Technical Report, Stanford University.

Ooms, L. M., Horan, K. A., Rahman, P., Seaton, G., Gurung, R. Kethesparan, D. S., \& Mitchell, C. A. (2009). The role of the inositol polyphosphate 5-phosphatases in cellular function and human disease. Biochemical Journal, 419(1), 29-49.

Ore, O. (1988). Number Theory and its History. New York: Dover.

Qiu, Y., Cheng, X., Ching, W-K., Jiang, H., \& Akutsu, T. (2015). On observability of attractors in Boolean networks. In Proceedings of 2015 IEEE International Conference on Bioinformatics and Biomedicine (pp. 263-266). NewYork: IEEE press.

Saadatpour, A., Albert, I., \& Albert, R. (2010).

Attractor analysis of asynchronous Boolean models of signal transduction networks. Journal of Theoretical Biology, 266, 641-656.

Samuelsson, B., \& Troein, C. (2003). Superpolynomial growth in the number of attractors in Kauffman networks. Physical Review Letters, 90, 098701.

Veliz-Cuba, A., Aguilar, B., Hinkelmann, F., \& Laubenbacher, R. (2014). Steady state analysis of Boolean molecular network models via model reduction and computational algebra. $B M C$ Bioinformatics, 15, 221.

Whitfield, M., George, L.K., Grant, G.D., \& Perou, C.M. (2006). Common markers of proliferation. Nature Reviews Cancer, 6 , 99-106.

Wu, X., Chen, L., \& Wang, X. (2014). Network biomarkers, interaction networks and dynamical network biomarkers in respiratory diseases. Clinical and Translational Medicine, 3, 16.

Yan, G., Tsekenis, G., Barzel, B., Liu, Y., Slotine, J., \& Barabàsi, A. (2015). Spectrum of controlling and observing complex networks. Nature Physics, 11, 779-786.

Zañudo, J.G.T., \& Albert, R. (2013). An effective network reduction approach to find the dynamical repertoire of discrete dynamic networks. Chaos, 23, 025111.
Zañudo, J.G.T., \& Albert, R. (2015). Cell fate reprogramming by control of intracellular network dynamics. PLoS Computational Biology, 11, e1004193.

Zhang, L., Reidy, S. P., Nicholson, T. E., Lee, H-J., Majdalawieh, A., Webber, C., Stewart, B. R, Dolphin, P., \& Ro, H-S. (2005). The role of AEBP1 in sex-specific diet-induced obesity. Molecular Medicine, 11, 39-47. 